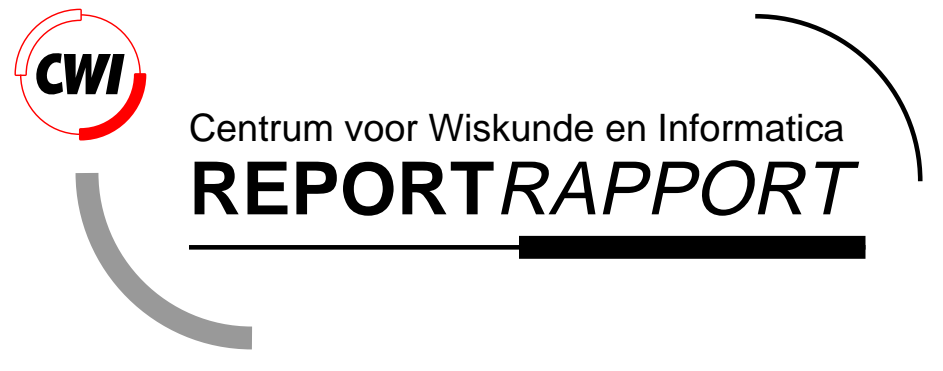

Bisimulation for probabilistic transition systems: a coalgebraic approach

E.P. de Vink, J.J.M.M. Rutten

Software Engineering (SEN)

SEN-R9825 October 1998 
Report SEN-R9825

ISSN 1386-369X

CWI

P.O. Box 94079

1090 GB Amsterdam

The Netherlands

CWI is the National Research Institute for Mathematics and Computer Science. CWI is part of the Stichting Mathematisch Centrum (SMC), the Dutch foundation for promotion of mathematics and computer science and their applications.

SMC is sponsored by the Netherlands Organization for Scientific Research (NWO). CWI is a member of ERCIM, the European Research Consortium for Informatics and Mathematics.

Copyright (C) Stichting Mathematisch Centrum P.O. Box 94079, 1090 GB Amsterdam (NL) Kruislaan 413, 1098 SJ Amsterdam (NL) Telephone +3120 5929333 Telefax +31205924199 


\title{
Bisimulation for Probabilistic Transition Systems: a coalgebraic approach
}

\author{
E.P. de Vink \\ Faculty of Mathematics and Computer Science, Vrije Universiteit \\ De Boelelaan 1081a, 1081 HV Amsterdam, The Netherlands \\ vink@cs.vu.nl \\ J.J.M.M. Rutten \\ $C W I$ \\ P.O. Box 94079, 1090 GB Amsterdam, The Netherlands \\ janr@cwi.nl
}

\begin{abstract}
The notion of bisimulation as proposed by Larsen and Skou for discrete probabilistic transition systems is shown to coincide with a coalgebraic definition in the sense of Aczel and Mendler in terms of a set functor, which associates to a set its collection of simple probability distributions. This coalgebraic formulation makes it possible to generalize the concepts of discrete probabilistic transition system and probabilistic bisimulation to a continuous setting involving Borel probability measures. A functor $\mathcal{M}_{1}$ is introduced that yields for a metric space its collection of Borel probability measures. Under reasonable conditions, this functor exactly captures generalized probabilistic bisimilarity. Application of the final coalgebra paradigm to a functor based on $\mathcal{M}_{1}$ then yields an internally fully abstract semantical domain with respect to probabilistic bisimulation, which is therefore well-suited for the interpretation of probabilistic specification and stochastic programming concepts.
\end{abstract}

1991 Mathematics Subject Classification: 68Q10, 68Q55

1991 Computing Reviews Classification System: D.3, F.1, F.3

Keywords 83 Phrases: Bisimulation, probabilistic transition systems, coalgebras, ultrametric spaces, Borel measures, final coalgebra

Note: This report is accepted for publication in TCS. An extended abstract of this report appeared in the proceedings of ICALP 1997, LNCS 1256, Springer-Verlag.

\section{Introduction}

For discrete probabilistic transition systems the notion of probabilistic bisimilarity of Larsen and Skou [LS91] is regarded as the basic process equivalence. Their definition was given for reactive systems. Subsequently, in [GSS95], Van Glabbeek, Smolka and Steffen give variations on the Larsen-Skou definition dealing with generative and so-called stratified systems. For a process language with probabilistic choice they prove a hierarchy-result on bisimulation for 
non-probabilistic, reactive, generative and stratified interpretations. Several other probabilistic equivalences are dealt with as well in the literature, e.g., more recently, [Hen95], in which decidability for finite bisimulations for hybrid automata is studied; [BLFG95], discussing specification and simulation for stochastic processes; [Seg95], on verification of randomized distributed algorithms; [BG96], dealing with performance analysis of concurrent systems; [KN96], presenting a concrete metric process domain for the modeling of probabilistic choice; and many more. (See the references in the papers mentioned.) In all papers, discrete probability distributions are used, and hence the transition systems that are treated are in essence of a finitely branching or image-finite nature.

For the exploration of probabilistic transition systems and stochastic equivalences in the setting of modeling continuous systems, such as real-time or hybrid systems, one usually wants to allow more general probability measures than the more limited discrete probability distributions. The papers [BDEP97, DEP98] are the single proposal into this direction that we know of. This approach is inspired by early work in [Gir81], on the one hand, and the recent development of open maps of [JNW96], on the other, and uses stochastic kernels over Polish spaces as transition systems and spans of zigzag morphism as bisimulations. They prove that their notion of bisimulation is an equivalence that agrees in the discrete case with the Larsen-Skou definition, but do not provide a characterization of bisimilarity in terms of transition steps, i.e., they do not give a continuous analogue for the Larsen-Skou bisimulation.

Here we attack the problem of continuous probabilistic transition systems and bisimulation by exploiting the transition-systems-as-coalgebras paradigm [RT93, Rut96, JR97]. Using a minimal amount of category theory (essentially the notions of category and functor), it can be summarized as follows: Let $\mathcal{F}: \mathcal{C} \rightarrow \mathcal{C}$ be any functor on a category $\mathcal{C}$. A coalgebra of $\mathcal{F}$ is an object $S$ in $\mathcal{C}$ together with an arrow $\alpha: S \rightarrow \mathcal{F}(S)$. It turns out that for many categories and functors, such a pair $(S, \alpha)$ represents a transition system, the type of which is determined by the functor $\mathcal{F}$. Vice versa, many types of transition systems can be captured by a functor this way. For instance, consider the familiar labeled transition systems $(S, A, \rightarrow)$, consisting of a set $S$ of states, a set $A$ of actions, and a transition relation $\rightarrow \subseteq S \times A \times S$ (cf. [Kel76, Plo81]). Put $\mathcal{L}(X)=\mathcal{P}(A \times X)$, the collection of all subsets of $A \times X$, for any set $X$, and, for $f: X \rightarrow Y$, define $\mathcal{L}(f): \mathcal{L}(X) \rightarrow \mathcal{L}(Y)$, by $\mathcal{L}(f)\left(\left\{\left(a_{i}, x_{i}\right) \mid i \in I\right\}\right)=\left\{\left(a_{i}, f\left(x_{i}\right)\right) \mid i \in I\right\}$. It can be easily shown that $\mathcal{L}$ is a functor on the category of sets and functions. A labeled transition system $(S, A, \rightarrow)$ can now be represented as an $\mathcal{L}$-coalgebra by defining

$$
\alpha: S \rightarrow \mathcal{L}(S), \quad s \mapsto\left\{\left(a, s^{\prime}\right) \mid\left(s, a, s^{\prime}\right) \in \rightarrow\right\} .
$$

Conversely, any $\mathcal{L}$-coalgebra corresponds to a transition system: If $(S, \alpha)$ is a coalgebra for $\mathcal{L}$, then $(S, A, \rightarrow)$, with $\rightarrow \subseteq S \times A \times S$ given by $\left(s, a, s^{\prime}\right) \in \rightarrow \Longleftrightarrow\left(a, s^{\prime}\right) \in \alpha(s)$, is clearly a transition system. (See Section 3 for more details.)

One of the advantages of the coalgebraic view on transition systems is the existence of a general definition of $\mathcal{F}$-bisimulation, for any functor $\mathcal{F}$ (cf. [AM89]). For instance, applying that definition to the functor $\mathcal{L}$ above yields the standard notion of strong bisimulation of [Mil80, Par81] (cf. Section 3). In general, the coalgebraic theory gives a generic approach to the definition and description of bisimulation: First define or characterize the transition systems one is interested in as coalgebras of a suitably chosen functor $\mathcal{F}$. Then obtain a definition of bisimulation for those systems by applying the categorical definition of $\mathcal{F}$-bisimulation.

The coalgebraic approach is applicable to various kinds of transition systems — see [Rut96] for many examples - including nondeterministic automata, infinite data structures, and object-based systems [Rei95, Jac96]. In the present paper, this scheme is used to describe 
discrete and continuous probabilistic transition systems and bisimulations. The functor $\mathcal{M}_{1}$, that is introduced in the sequel, assigns to an ultrametric space its collection of Borel probability measures. It is shown that the corresponding notion of $\mathcal{M}_{1}$-bisimulation coincides, under mild conditions, with the continuous analogue of Larsen-Skou bisimulation. This extends a similar result for the discrete case, which is in fact given first: the functor $\mathcal{D}$, which assigns to a set the collection of its simple probability distributions, is shown to yield a categorical characterization of Larsen-Skou bisimulation. Hence, in agreement with general opinion, also from the coalgebraic point of view the latter equivalence is suggested as the canonical one.

Another appealing aspect of the coalgebraic approach is a canonical way of finding internally fully abstract domains of bisimulation, where two elements are equal if and only if they are bisimilar. It follows from a simple but very general argument that final coalgebras are fully abstract (see Aczel's final coalgebra model for nonwellfounded sets [Acz88], and also [RT93]). Here, final means that there exists a unique homomorphism from any coalgebra to the final one. (One can argue that finality is to the world of coalgebras what initiality is to the world of algebras, cf. [MG85].) We shall show that it follows from general coalgebraic considerations [AR89, Bar93, RT93] that both functors that are considered have a final coalgebra, which consequently is internally fully abstract with respect to (discrete and continuous) probabilistic bisimulation. Therefore these final coalgebras can be exploited as semantic domains for probabilistic bisimulation (an important direction for future research).

As mentioned above, the functor $\mathcal{M}_{1}$ is defined on ultrametric spaces, and the Borel $\sigma$ algebras and associated measures are taken with respect to the metric topology. Our reasons for considering metric spaces rather than the, in semantical contexts, more standard use of ordered structures, such as [Jon89, JP89] and [Eda95a, Eda95b], are twofold. Firstly, one can resort to the rich literature on standard measure theory for metric spaces (see, e.g., [KV84]). Secondly, we can use the recently developed coalgebraic theory on metric spaces [AR89, RT94], which seems to be better suited to describe (both ordinary and probabilistic) bisimulation than the corresponding theory for ordered spaces (cf. [RT94]). We shall see that the functor involved is locally contractive, from which it follows that it has a final coalgebra. Because of the coalgebraic definition of bisimulation, we thus obtain an internally fully abstract domain. Such a full abstractness result has been lacking so far in the literature.

In conclusion, $\mathcal{D}$-bisimilarity and Larsen-Skou bisimilarity coincide for discrete probabilistic transition systems. For the continuous case, the functor $\mathcal{M}_{1}$ captures the generalization of probabilistic transition systems, and - under conditions - characterizes the associated notion of probabilistic bisimulation. In both settings a final coalgebra and hence, internally fully abstract domain exists, which can be exploited in the construction of domains for probabilistic bisimulation semantics.

Acknowledgments We are grateful to Jaco de Bakker, Henno Brandsma, Franck van Breugel, Prakash Panangaden, the anonymous referees, and, as always, the members of the Amsterdam Concurrency Group for discussions and comments on this paper.

\section{Mathematical Preliminaries}

Basic measure theoretic definitions (See, e.g., the standard textbook [Rud66].) A $\sigma$-algebra $\Sigma$ on a set $X$ is a collection of subsets which contains $X$ and is closed under complement and countable union. Elements $E$ of $\Sigma$ are called measurable subsets of $X$. Trivially, the powerset $\mathcal{P}(X)$ is a $\sigma$-algebra for $X$. If $X$ is a topological space, the Borel $\sigma$-algebra $\mathcal{B}(X)$ is defined 
as the least $\sigma$-algebra containing all open sets.

A function $\mu: \Sigma \rightarrow[0,1]$, where $\Sigma$ is a $\sigma$-algebra on a set $X$, is called a $\Sigma$-probability measure if $\mu(X)=1$ and $\mu$ is $\sigma$-additive, i.e., $\mu\left(\bigcup_{i \in I} E_{i}\right)=\sum_{i \in I} \mu\left(E_{i}\right)$ for any countable disjoint collection of measurable sets $\left\{E_{i} \mid i \in I\right\}$. For $X$ a topological space, a Borel probability measure is a probability measure on $X$ taken with respect to the Borel $\sigma$-algebra $\mathcal{B}(X)$. For $x \in X$, the Dirac-measure $\delta_{x}$ is given by $\delta_{x}(E)=1$ if $x \in E$, and $\delta_{x}(E)=0$ otherwise. A function $\mu: X \rightarrow[0,1]$ is called a simple probability distribution if there exist $n$ distinct points $x_{1}, \ldots, x_{n}, n>0$, such that $\mu\left(x_{1}\right)+\cdots+\mu\left(x_{n}\right)=1$ and $\mu(x)=0$ for $x \notin\left\{x_{1}, \ldots, x_{n}\right\}$. The set $\left\{x_{1}, \ldots, x_{n}\right\}$ is called the support of $\mu$. $\mathcal{D}(X)$ denotes the collection of all simple probability distributions on $X$. For $E \subseteq X, \mu[E]$ is short for $\sum_{x \in E} \mu(x)$. This way, a simple probability distribution corresponds to a convex linear combination of Dirac-measures.

Metric spaces (See, e.g., the monograph [BV96].) A pair $(M, d)$ with $M$ a nonempty set and $d: M^{2} \rightarrow[0,1]$ is called an ultrametric space if, for all $x, y, z \in M: d(x, y)=d(y, x)$, $d(x, y)=0 \Leftrightarrow x=y$, and $d(x, z) \leq \max \{d(x, y), d(y, z)\}$. The last expression is referred to as the strong triangle inequality. For metric spaces $M_{1}, M_{2}$, a function $f: M_{1} \rightarrow M_{2}$ is called nonexpansive if $d_{2}(f(x), f(y)) \leq d_{1}(x, y)$, for all $x, y \in M$. The function $f$ is called $\kappa$-contractive in case $d_{2}(f(x), f(y)) \leq \kappa \cdot d_{1}(x, y)$, for all $x, y \in M$, where $\kappa$ is a constant with $0 \leq \kappa<1$. The collection of all nonexpansive mappings from $M_{1}$ to $M_{2}$ is denoted by $M_{1} \rightarrow_{1} M_{2}$. We use the notation $\mathcal{O}$, or more explicit $\mathcal{O}(M)$, for the collection of all open subsets of $M$.

Binary relations For a binary relation $R \subseteq S \times T$ we use $\pi_{1}$ and $\pi_{2}$ for the projections of $R$ on $S$ and $T$, respectively. $R$ is called total if the two projections $\pi_{1}$ and $\pi_{2}$ are surjective. We say that $R$ is z-closed if, for all $s, s^{\prime} \in S, t, t^{\prime} \in T, R(s, t) \wedge R\left(s^{\prime}, t\right) \wedge R\left(s^{\prime}, t^{\prime}\right) \Rightarrow R\left(s, t^{\prime}\right)$. If we put, for $n \in \mathbb{N}, R_{0}=R, R_{n+1}=\left\{\left(s, t^{\prime}\right) \in S \times T \mid \exists s^{\prime} \in S, t \in T: R(s, t) \wedge R_{n}\left(s^{\prime}, t\right) \wedge\right.$ $R\left(s^{\prime}, t^{\prime}\right)$ \}, and $R^{*}=\bigcup_{n \in \mathbb{N}} R_{n}$, we have that $R^{*}$ is the least z-closed binary relation on $S \times T$ containing $R$. (Note that $R_{n}(s, t)$ iff $\exists s_{0}, \ldots, s_{n}, t_{0}, \ldots, t_{n}: s_{0}=s \wedge t_{n}=t \wedge \forall i \leq n: R\left(s_{i}, t_{i}\right) \wedge$ $\forall i<n: R\left(s_{i+1}, t_{i}\right)$.) Below we will employ, for $s \in S$, the notation $F(s)=\{t \in T \mid R(s, t)\}$ and, for $U \subseteq S, F[U]=\bigcup_{s \in U} F(s)$, and, likewise, for $t \in T, E(t)=\{s \in S \mid R(s, t)\}$, and, for $V \subseteq T, E[V]=\bigcup_{t \in V} E(t)$. Please note the different usage of brackets in $E[\cdot]$ and $F[\cdot]$ yielding subsets of $S$ and $T$, compared to the notation $\mu[\cdot]$, introduced above, yielding, for a simple probability distribution $\mu$, a real number.

\section{Coalgebras}

We briefly recall the basic notions and facts of coalgebra, which is a general theory of (transition and dynamical) systems, phrased in the language of category theory. For an overview of the theory of coalgebras, containing many references and examples, see [Rut96].

Let $\mathcal{C}$ be either the category of sets and functions, or the category of ultrametric spaces and nonexpansive mappings. (These are the only categories playing a role in this paper.) Let $\mathcal{F}: \mathcal{C} \rightarrow \mathcal{C}$ be a functor. An $\mathcal{F}$-coalgebra is a pair $(S, \alpha)$ consisting of an object $S$ in $\mathcal{C}$ together with an arrow $\alpha: S \rightarrow \mathcal{F}(S)$ in $\mathcal{C}$, called a coalgebra structure on $S$. A homomorphism between two $\mathcal{F}$-coalgebras $(S, \alpha)$ and $(T, \beta)$ is an arrow $f: S \rightarrow T$ in $\mathcal{C}$ such that $\mathcal{F}(f) \circ \alpha=\beta \circ f$.

An $\mathcal{F}$-bisimulation between two $\mathcal{F}$-coalgebras $(S, \alpha)$ and $(T, \beta)$ is a relation $R \subseteq S \times T$ for which there exists a coalgebra structure $\gamma: R \rightarrow \mathcal{F}(R)$ such that the projections $\pi_{1}: R \rightarrow S$ 
and $\pi_{2}: R \rightarrow T$ are homomorphisms: $\alpha \circ \pi_{1}=\mathcal{F}\left(\pi_{1}\right) \circ \gamma$ and $\beta \circ \pi_{2}=\mathcal{F}\left(\pi_{2}\right) \circ \gamma$. We then say that $R$ is an $\mathcal{F}$-bisimulation for $\alpha$ and $\beta$. The arrow $\gamma$ is called mediating for $\alpha$ and $\beta$. We write $x \sim y$ (' $x$ and $y$ are $\mathcal{F}$-bisimilar') whenever there exists an $\mathcal{F}$-bisimulation $R$ with $(x, y) \in R$.

$\begin{array}{ccccr}S & \pi_{1} & R & \pi_{2} & T \\ \alpha & & \gamma & & \beta \\ \mathcal{F}(S) & \mathcal{F}\left(\pi_{1}\right) & \mathcal{F}(R) & \mathcal{F}\left(\pi_{2}\right) & \mathcal{F}(T)\end{array}$

Example Let us elaborate the above definitions for the example of the functor $\mathcal{L}$ presented in the introduction. Let Set be the category of sets and functions. For a fixed set $A$, the functor $\mathcal{L}:$ Set $\rightarrow$ Set was given by $\mathcal{L}(X)=\mathcal{P}(A \times X)$, and $\mathcal{L}(f)(V)=\{(a, f(x)) \mid(a, x) \in V\}$, for a set $X, f: X \rightarrow Y$, and $V \in \mathcal{P}(A \times X)$.

Let $(S, \alpha)$ and $(T, \beta)$ be two $\mathcal{L}$-coalgebras, so $\alpha: S \rightarrow \mathcal{L}(S)$ and $\beta: T \rightarrow \mathcal{L}(T)$. Below we will use a more suggestive notation like $s \stackrel{a}{\rightarrow}_{\alpha} s^{\prime}$ instead of $\left(a, s^{\prime}\right) \in \alpha(s)$. By definition, a function $f: S \rightarrow T$ is an $\mathcal{L}$-coalgebra homomorphism iff it satisfies $\mathcal{L}(f) \circ \alpha=\beta \circ f$, that is

$$
\left\{\left(a, f\left(s^{\prime}\right)\right) \mid s \stackrel{a}{\rightarrow}_{\alpha} s^{\prime}\right\}=\left\{\left(a, t^{\prime}\right) \mid f(s) \stackrel{a}{\rightarrow}_{\beta} t^{\prime}\right\},
$$

for all $s \in S$. Equivalently,

$$
s \stackrel{a}{\rightarrow}_{\alpha} s^{\prime} \Rightarrow f(s) \stackrel{a}{\rightarrow}_{\beta} f\left(s^{\prime}\right), \text { and } f(s) \stackrel{a}{\rightarrow}_{\beta} t^{\prime} \Rightarrow \exists s^{\prime} \in S: s \stackrel{a}{\rightarrow}_{\alpha} s^{\prime} \wedge f\left(s^{\prime}\right)=t^{\prime} .
$$

Next consider an $\mathcal{L}$-bisimulation $R \subseteq S \times T$ for $(S, \alpha)$ and $(T, \beta)$, with coalgebra structure $\gamma: R \rightarrow \mathcal{L}(R)$. Then $\mathcal{L}\left(\pi_{1}\right) \circ \gamma=\alpha \circ \pi_{1}$ and $\mathcal{L}\left(\pi_{2}\right) \circ \gamma=\beta \circ \pi_{2}$ are equivalent with

$$
\begin{aligned}
& (s, t) \stackrel{a}{\rightarrow} \gamma\left(s^{\prime}, t^{\prime}\right) \Rightarrow s \stackrel{a}{\rightarrow} \alpha s^{\prime} \text { and } s \stackrel{a}{\rightarrow} \alpha s^{\prime} \Rightarrow \exists t^{\prime} \in T:(s, t) \stackrel{a}{\rightarrow} \gamma\left(s^{\prime}, t^{\prime}\right), \\
& (s, t) \stackrel{a}{\rightarrow}_{\gamma}\left(s^{\prime}, t^{\prime}\right) \Rightarrow t \stackrel{a}{\rightarrow} \beta t^{\prime} \text { and } t \stackrel{a}{\rightarrow}_{\beta} t^{\prime} \Rightarrow \exists s^{\prime} \in S:(s, t) \stackrel{a}{\rightarrow}_{\gamma}\left(s^{\prime}, t^{\prime}\right),
\end{aligned}
$$

for all $(s, t) \in R$. It follows that if $R(s, t)$ then

$$
s \stackrel{a}{\rightarrow} \alpha s^{\prime} \Rightarrow \exists t^{\prime} \in T: R\left(s^{\prime}, t^{\prime}\right) \wedge t \stackrel{a}{\rightarrow} \beta t^{\prime} \text { and } t \stackrel{a}{\rightarrow}_{\beta} t^{\prime} \Rightarrow \exists s^{\prime} \in S: R\left(s^{\prime}, t^{\prime}\right) \wedge s \stackrel{a}{\rightarrow}_{\alpha} s^{\prime} .
$$

This is precisely the familiar notion of strong bisimilarity for labeled transition systems of [Mil80, Par81]. Thus $R$ is a strong bisimulation for $(S, \alpha)$ and $(T, \beta)$. Conversely, if $R \subseteq S \times T$ is a strong bisimulation for $(S, \alpha)$ and $(T, \beta)$, we define $\gamma: R \rightarrow \mathcal{L}(R)$, for $(s, t)$ and $\left(s^{\prime}, t^{\prime}\right)$ in $R$, by

$$
(s, t) \stackrel{a}{\rightarrow}_{\gamma}\left(s^{\prime}, t^{\prime}\right) \Longleftrightarrow s \stackrel{a}{\rightarrow}_{\alpha} s^{\prime} \wedge t \stackrel{a}{\rightarrow}_{\beta} t^{\prime}
$$

It can be easily proved that $R$ is a $\mathcal{L}$-bisimulation for $(S, \alpha)$ and $(T, \beta)$ with mediating function $\gamma$. (More details, and many more examples, can be found in [Rut96].)

An $\mathcal{F}$-coalgebra $(D, \delta)$ is called final if there exists for any $\mathcal{F}$-coalgebra $(S, \alpha)$ a unique homomorphism from $(S, \alpha)$ to $(D, \delta)$. We have the following result.

Theorem 3.1 (Internal full abstractness) For a final $\mathcal{F}$-coalgebra $(D, \delta)$ and $x, y \in D, x=y$ if and only if $x \sim y$. 
The proof is easy, see [RT93] or [Rut96, Thm 9.2]. The main difficulty in obtaining full abstractness lies in the construction of a final coalgebra, which in general is non-trivial.

Functors $\mathcal{F}$ that preserve weak pullbacks, a categorical property discussed in the Appendix, are particularly well behaved. For such functors, unions and relational compositions of $\mathcal{F}$ bisimulations yield $\mathcal{F}$-bisimulations again. As a consequence, bisimilarity is an equivalence relation. There is also the following generalization of Theorem 3.1.

Theorem 3.2 (Full abstractness) Let $\mathcal{F}$ be a functor preserving weak pullbacks, $(S, \alpha)$ an $\mathcal{F}$-coalgebra, $(D, \delta)$ a final $\mathcal{F}$-coalgebra, and $f: S \rightarrow D$ the unique homomorphism from $(S, \alpha)$ to $(D, \delta)$. For any $x, y \in S, f(x)=f(y)$ if and only if $x \sim y$.

The proof of Theorem 3.2 is again simple, and can be found in [RT93] or [Rut96, Thm 9.3]. The same applies to the properties of $\mathcal{F}$-bisimulations mentioned above, which are proved (for functors on Set) in [Rut96, Thm 5.4 and Thm 5.5].

\section{A coalgebraic interpretation of Larsen-Skou bisimulation}

Starting from the definitions of a discrete probabilistic transition system and probabilistic bisimulation as proposed in the literature, we will consider generalizations of discrete probabilistic transition systems as coalgebras of a functor $\mathcal{D}$ on the category Set of sets and functions. We will show that $\mathcal{D}$-bisimilarity implies probabilistic bisimilarity, and, using the notion of z-closure, that probabilistic bisimulation and totality imply $\mathcal{D}$-bisimilarity. Furthermore it is shown how this leads to the existence of a fully abstract domain.

Definition 4.1 [LS91, GSS95] A discrete probabilistic transition system is a tuple $(\operatorname{Pr}$, Act, $\mu)$ where $\operatorname{Pr}$ is a nonempty set of processes, Act is a given set of actions, and the mapping $\mu: \operatorname{Pr} \times \operatorname{Act} \times \operatorname{Pr} \rightarrow[0,1]$ is a so-called transition probability function, i.e., $\mu(P, a, \cdot)$ is either the zero-map, or a simple probability distribution, for all $P \in \operatorname{Pr}, a \in$ Act.

A probabilistic bisimulation for a discrete probabilistic transition system is an equivalence '”' on $\operatorname{Pr}$ such that

$$
P \equiv Q \Rightarrow \mu[P, a, E]=\mu[Q, a, E],
$$

for all $P, Q \in \operatorname{Pr}, a \in A c t$, and equivalence classes $E \in \operatorname{Pr} / \equiv$. Two processes $P$ and $Q$ are said to be probabilistic bisimilar if some probabilistic bisimulation contains the pair $(P, Q)$.

In Section 2 we introduced the notation $\mathcal{D}(S)$ for the collection of all simple probability distributions over a set $S$. In fact, $\mathcal{D}$ can be extended to a Set-functor by defining, for a mapping $f: S \rightarrow T$, a function $\mathcal{D}(f): \mathcal{D}(S) \rightarrow \mathcal{D}(T)$ which maps a simple distribution $\mu$ on $S$ to $\mathcal{D}(f)(\mu)$ defined, for $t \in T$, by $\mathcal{D}(f)(\mu)(t)=\mu\left[f^{-1}(\{t\})\right]$.

Let 0 represent termination. Note that a probabilistic transition system is just a mapping $\mu: \operatorname{Pr} \times A c t \rightarrow \mathcal{D}(\operatorname{Pr})+\{\mathbf{0}\}$ or, equivalently, a function

$$
\mu: \operatorname{Pr} \rightarrow(A c t \rightarrow(\mathcal{D}(\operatorname{Pr})+\{\mathbf{0}\})) .
$$

In other words, a probabilistic transition system is precisely a coalgebra of the functor Act $\rightarrow(\mathcal{D}(\cdot)+\{\mathbf{0}\})$. Applying the category theoretical machinery as described in Section 3 now gives us the coalgebraic notion of bisimulation. We will show that it corresponds to 
(actually generalizes) the notion of probabilistic bisimulation of Definition 4.1, thus providing categorical evidence for the Larsen-Skou bisimulation as the canonical process equivalence for discrete probabilistic transition systems.

For clarity of presentation we suppress, for the moment, the action component of a probabilistic transition system, and also do not bother about termination. Thus we consider coalgebras of the functor $\mathcal{D}$ itself. (As a consequence, in the sense of Definition 4.2, the relation $S \times T$ is always a bisimiulation for $\mathcal{D}$.) It turns out that, the presence of labels and termination does not make any essential difference for the technical content of what follows. Before we relate probabilistic bisimulation with $\mathcal{D}$-bisimulation, we first give a generalization of Definition 4.1, by allowing bisimulations between different transition systems, which are not necessarily equivalence relations.

Definition 4.2 Let $\alpha: S \rightarrow \mathcal{D}(S), \beta: T \rightarrow \mathcal{D}(T)$ be two (stripped) discrete probabilistic transition systems. A binary relation $R \subseteq S \times T$ is called a probabilistic bisimulation for $\alpha, \beta$ iff $R(s, t) \Rightarrow \alpha(s)[U]=\beta(t)[V]$, for all $s \in S, t \in T$ and $U \subseteq S, V \subseteq T$ such that $\pi_{1}^{-1}(U)=\pi_{2}^{-1}(V)$. Two elements $s \in S, t \in T$ are said to be probabilistic bisimilar if some probabilistic bisimulation contains the pair $(s, t)$.

Note that if $R$ is an equivalence relation, then $\pi_{1}^{-1}(U)=\pi_{2}^{-1}(V)$ if and only if $U=\bigcup_{i \in I} E_{i}=$ $V$, for some collection of equivalence classes $\left\{E_{i} \mid i \in I\right\}$ of $R$. Thus in this case, the condition on $U$ and $V$ in Definition 4.2 amounts to the assumption of $E$ being an equivalence class in Definition 4.1, or, following the terminology of [Hen95], $U$ and $V$ are the same 'E'-block. This shows that Definition 4.1 is a special instance of Definition 4.2 ('modulo' the presence of labels and termination).

Next we relate the notion of $\mathcal{D}$-bisimulation to the notion of probabilistic bisimulation of Definition 4.2. Recall, from Section 3, that a homomorphism for two $\mathcal{D}$-coalgebras $(S, \alpha)$, $(T, \beta)$ is a function $f: S \rightarrow T$ such that $\mathcal{D}(f) \circ \alpha=\beta \circ f$. Also recall that a $\mathcal{D}$-bisimulation is a relation $R \subseteq S \times T$ together with a mapping $\gamma: R \rightarrow \mathcal{D}(R)$ such that $\mathcal{D}\left(\pi_{1}\right) \circ \gamma=\alpha \circ \pi_{1}$ and $\mathcal{D}\left(\pi_{2}\right) \circ \gamma=\beta \circ \pi_{2}$. More specifically, for $(s, t) \in R$ it holds that $\gamma(s, t)\left[\pi_{1}^{-1}\left(\left\{s^{\prime}\right\}\right)\right]=$ $\alpha\left(\pi_{1}(s, t)\right)\left(s^{\prime}\right)=\alpha(s)\left(s^{\prime}\right)$, and, symmetrically, $\gamma(s, t)\left[\pi_{2}^{-1}\left(\left\{t^{\prime}\right\}\right)\right]=\beta(t)\left(t^{\prime}\right)$, for all $s^{\prime} \in S$, $t^{\prime} \in T$.

Lemma 4.3 Let $\alpha: S \rightarrow \mathcal{D}(S)$ and $\beta: T \rightarrow \mathcal{D}(T)$ be two discrete probabilistic transition systems. Let $R$ be a D-bisimulation for $\alpha, \beta$. Then $R$ is a probabilistic bisimulation for $\alpha, \beta$.

Proof Let $\gamma: R \rightarrow \mathcal{D}(R)$ be mediating for $\alpha, \beta$, i.e., $\alpha \circ \pi_{1}=\mathcal{D}\left(\pi_{1}\right) \circ \gamma$ and $\beta \circ \pi_{2}=\mathcal{D}\left(\pi_{2}\right) \circ \gamma$. Suppose $R(s, t)$ and $\pi_{1}^{-1}(U)=\pi_{2}^{-1}(V)$. We then have

$$
\begin{aligned}
\alpha(s)[U] & =\alpha\left(\pi_{1}(s, t)\right)[U]=\mathcal{D}\left(\pi_{1}\right)(\gamma(s, t))[U]=\gamma(s, t)\left[\pi_{1}^{-1}(U)\right]= \\
& \stackrel{(*)}{=} \gamma(s, t)\left[\pi_{2}^{-1}(V)\right]=\mathcal{D}\left(\pi_{2}\right)(\gamma(s, t))[V]=\beta\left(\pi_{2}(s, t)\right)[V]=\beta(t)[V]
\end{aligned}
$$

exploiting the definitions of $\mathcal{D}\left(\pi_{1}\right), \mathcal{D}\left(\pi_{2}\right)$, and at $(*)$ the assumption on $U$ and $V$.

The reverse of the above lemma is more intricate. We will first use the concept of z-closure and associated properties as developed in Section 2.

Lemma 4.4 If $R \subseteq S \times T$ is a probabilistic bisimulation for $\alpha: S \rightarrow \mathcal{D}(S), \beta: T \rightarrow \mathcal{D}(T)$, then so is $R^{*}$, the $z$-closure of $R$. 
Proof We have, by definition, $R^{*}=\bigcup\left\{R_{n} \mid n \in \mathbb{N}\right\}$ with $R_{n}$ as in Section 2. Therefore the lemma directly follows from the implication

$$
R_{n}(s, t) \Rightarrow \alpha(s)[U]=\beta(t)[V]
$$

for $n \in \mathbb{N}$ (and $U, V$ such that $\pi_{1}^{-1}(U)=\pi_{2}^{-1}(V)$ ), which can be shown by induction on $n$ : [0] Clearly, $R_{0}=R$ by definition and $R$ is a probabilistic bisimulation. $[n+1]$ Suppose $R_{n+1}(s, t)$. Pick $t^{\prime} \in T, s^{\prime} \in S$ such that $R\left(s, t^{\prime}\right), R_{n}\left(s^{\prime}, t^{\prime}\right)$ and $R\left(s^{\prime}, t\right)$. We then have $\alpha(s)[U]=\beta\left(t^{\prime}\right)[V]$, $\alpha\left(s^{\prime}\right)[U]=\beta\left(t^{\prime}\right)[V]$ and $\alpha\left(s^{\prime}\right)[U]=\beta(t)[V]$, since $R$ is a probabilistic bisimulation and by the induction hypothesis. So $\alpha(s)[U]=\beta(t)[V]$, which was to be shown.

So, if $s \in S$ and $t \in T$ are probabilistic bisimilar, we can assume - without loss of generalitythat there exists a z-closed probabilistic bisimulation containing $(s, t)$. We will need, for technical reasons, that $R$ is total. This is implied by the common assumption of transition systems to have a distinguished initial state and considering reachable states only.

Theorem 4.5 Let $R \subseteq S \times T$ be a probabilistic bisimulation for $\alpha: S \rightarrow \mathcal{D}(S)$ and $\beta: T \rightarrow$ $\mathcal{D}(T)$. Moreover, assume $R$ to be z-closed and total. Then $R$ is a $\mathcal{D}$-bisimulation.

Proof Let $(s, t) \in R$. We define $\gamma: R \rightarrow \mathcal{D}(R)$ as follows. Let $\gamma_{s, t}$ be short for $\gamma(s, t)$, and put

$$
\gamma_{s, t}\left(s^{\prime}, t^{\prime}\right)=\left\{\begin{array}{cl}
0 & \text { if } \beta(t)\left[F\left(s^{\prime}\right)\right]=0 \\
\frac{\alpha(s)\left(s^{\prime}\right) \cdot \beta(t)\left(t^{\prime}\right)}{\beta(t)\left[F\left(s^{\prime}\right)\right]} & \text { otherwise. }
\end{array}\right.
$$

(The notation $F(s)$ was introduced in Section 2: $F(s)=\{t \in T \mid R(s, t)\}$. Also $E(t)=\{s \in$ $S \mid R(s, t)\}$.) By z-closedness of $R$ it follows from $R\left(s^{\prime}, t^{\prime}\right)$ that $\pi_{1}^{-1}\left(E\left(t^{\prime}\right)\right)=\pi_{2}^{-1}\left(F\left(s^{\prime}\right)\right)$ and hence $\alpha(s)\left[E\left(t^{\prime}\right)\right]=\beta(t)\left[F\left(s^{\prime}\right)\right]$. Therefore the above definition of $\gamma_{s, t}$ is symmetric in $s$ and $t$. We will check that $\gamma_{s, t}$ is a simple probability distribution. First we claim

$$
\gamma_{s, t}\left[\left\{s^{\prime}\right\} \times F\left(s^{\prime}\right)\right]=\alpha(s)\left(s^{\prime}\right) .
$$

This can be seen as follows: By z-closedness of $R$ we have $\pi_{1}^{-1}\left(E\left[F\left(s^{\prime}\right)\right]\right)=\pi_{2}^{-1}\left(F\left(s^{\prime}\right)\right)$. We distinguish two cases: Suppose $\beta(t)\left[F\left(s^{\prime}\right)\right]=0$. Then, by definition, $\gamma_{s, t}\left[\left\{s^{\prime}\right\} \times F\left(s^{\prime}\right)\right]=0$, and, since $R$ is a probabilistic bisimulation it holds that

$$
0 \leq \alpha(s)\left(s^{\prime}\right) \leq \alpha(s)\left[E\left[F\left(s^{\prime}\right)\right]\right]=\beta(t)\left[F\left(s^{\prime}\right)\right]=0 .
$$

(Totality of $R$ implies $s^{\prime} \in E\left[F\left(s^{\prime}\right)\right]$.) So it follows that $\alpha(s)\left(s^{\prime}\right)=0$. Now suppose $\beta(t)\left[F\left(s^{\prime}\right)\right] \neq 0$. Then we have

$$
\begin{aligned}
& \gamma_{s, t}\left[\left\{s^{\prime}\right\} \times F\left(s^{\prime}\right)\right] \\
& =\sum_{t^{\prime \prime} \in F\left(s^{\prime}\right)} \frac{\alpha(s)\left(s^{\prime}\right) \cdot \beta(t)\left(t^{\prime \prime}\right)}{\beta(t)\left[F\left(s^{\prime}\right)\right]} \\
& =\frac{\alpha(s)\left(s^{\prime}\right) \cdot \beta(t)\left[F\left(s^{\prime}\right)\right]}{\beta(t)\left[F\left(s^{\prime}\right)\right]} \\
& =\alpha(s)\left(s^{\prime}\right) .
\end{aligned}
$$


This proves the claim. By equation (4.1) we have $\gamma_{s, t}[R]=\sum_{s^{\prime} \in S} \gamma_{s, t}\left[\left\{s^{\prime}\right\} \times F\left(s^{\prime}\right)\right]=$ $\sum_{s^{\prime} \in S} \alpha(s)\left(s^{\prime}\right)=1$.

We check that $\gamma$ is mediating for $\alpha, \beta$. The equality $\alpha \circ \pi_{1}=\mathcal{D}\left(\pi_{1}\right) \circ \gamma$ is a direct consequence of (4.1). For showing $\beta \circ \pi_{2}=\mathcal{D}\left(\pi_{2}\right) \circ \gamma$ a similar argument can be applied using the property $\gamma_{s, t}\left[E\left(t^{\prime}\right) \times\left\{t^{\prime}\right\}\right]=\beta(t)\left(t^{\prime}\right)$. Conclusion: $R$ is a $\mathcal{D}$-bisimulation.

The format of the definition of $\gamma(s, t)$ is reminiscent of the discrete probability distributions of [JL91]. It is however not clear how their notion of probabilistic specification extends to the continuous setting of Section 5 .

It is straightforward to adapt the above line of reasoning to a functor $\mathcal{D}^{\prime}$ given by $\mathcal{D}^{\prime}=$ Act $\rightarrow(\mathcal{D}(\cdot)+\{\mathbf{0}\})$. The discrete probabilistic transition systems of Definition 4.1 are in 1-1 correspondence with the coalgebras of this functor, and the notion of $\mathcal{D}^{\prime}$-bisimulation coincides with that of probabilistic bisimulation of Definition 4.1 (for total relations $R$ ).

We can now benefit from some general insights in the theory of coalgebras, by applying (a minor variation on) a result from Barr.

Theorem 4.6 The functor $\mathcal{D}^{\prime}$ (and also $\mathcal{D}$ ) has a final coalgebra.

Proof Let $\alpha: S \rightarrow \mathcal{D}^{\prime}(S)$ be a $\mathcal{D}^{\prime}$-coalgebra, and $s$ an element in $S$. The subcoalgebra generated by $s$, denoted by $\langle s\rangle$, is defined as the smallest subcoalgebra of $(S, \alpha)$ containing $s$. It can be constructed by starting with the set $\{s\}$, then adding the finitely many elements $s^{\prime}$ for which $\alpha(s)(a)\left(s^{\prime}\right)$ is strictly positive (note that $\alpha(s)(a)$ is a simple probability distribution), and so on. Assuming that the set Act is countable, it follows that at each of the countably many stages of this construction, only countably many elements are added. Thus the set $\langle s\rangle$ is countable. Since $(S, \alpha)$ and $s$ were arbitrary, we have proved that the functor $\mathcal{D}^{\prime}$ is bounded: the size of any $\mathcal{D}^{\prime}$-coalgebra generated by a singleton is bounded (by the cardinal number $\omega$ ).

Now we can apply a general result from the theory of coalgebras, which is proved in [Rut96, Thm 10.4], based on a result of [Bar93]. It simply states that any bounded functor has a final coalgebra. Since $\mathcal{D}^{\prime}$ is bounded, it has a final coalgebra.

The final coalgebra for $\mathcal{D}^{\prime}$ is nontrivial. The final coalgebra for $\mathcal{D}$, though, is degenerate: it equals the one element set. Due to the absence of labels and a concept of termination as present for $\mathcal{D}^{\prime}$, all elements in any two $\mathcal{D}$-coalgebras are probabilistically bisimilar.

Let $\mathbb{P}$ be the final $\mathcal{D}^{\prime}$-coalgebra, so $\mathbb{P} \cong A c t \rightarrow(\mathcal{D}(\mathbb{P})+\{\mathbf{0}\})$. (Note that final coalgebras are always fixed points. See, e.g., [Rut96, Thm 9.1].) The following is now immediate by Theorem 3.1.

Corollary 4.7 The system $\mathbb{P}$ is internally fully abstract with respect to the original notion of probabilistic bisimulation of Definition 4.1.

The functor $\mathcal{D}$ preserves weak pullbacks, which is proved in the Appendix. As an immediate consequence, also the functor $\mathcal{D}^{\prime}$ does. Therefore, probabilistic transition systems and bisimulations are well behaved: unions and compositions are bisimulations again, and the bisimilarity relation is an equivalence relation. Furthermore, there is the following instantiation of Theorem 3.2 .

Theorem 4.8 (Full abstractness) Let $(S, \alpha)$ be a discrete probabilistic transition system, and $f: S \rightarrow \mathbb{P}$ the unique homomorphism from $S$ to $\mathbb{P}$. For any $x, y \in S, f(x)=f(y)$ if and only if $x \sim y$. 


\section{$5 \quad \mathcal{M}_{1}$-Bisimilarity for Probabilistic Transition Systems}

The previous section illustrates that in a discrete probabilistic setting, a coalgebraic interpretation of probabilistic transition systems and bisimulation can be given, which reflects the usual 'direct' approach. One of the advantages of the abstract coalgebraic approach is that it can fairly easily be generalized to the continuous setting of stochastic systems. We will now, in fact, allow probability measures to play the role of the simple distributions in the definition of a probabilistic transition system.

Probability measures only make sense in the context of a $\sigma$-algebra. When the collection $\operatorname{Pr}$ of processes comes equipped with a topology — as is the case if the set of processes is endowed with an order or a metric structure - the natural choice for this $\sigma$-algebra is the Borel $\sigma$ algebra $\mathcal{B}(\operatorname{Pr})$, i.e. the least $\sigma$-algebra containing all the open sets of processes. As mentioned in the introduction, we use ultrametric spaces because of a combination of the following two reasons: the technical advantage of a close relationship between standard measure theory and metric topology, and the availability of a final coalgebra theorem in the metric setting, leading to a fully abstract domain for general probabilistic bisimulation. (We note that the second point would also apply to the order-theoretic case. See [RT93].)

The generalization of the notion of a discrete probabilistic transition system and the associated concept of bisimulation as proposed by Larsen and Skou is as follows.

Definition 5.1 A (general) probabilistic transition system is a tuple $(\operatorname{Pr}, A c t, \mu)$ where $\operatorname{Pr}$ is any ultrametric space of processes, Act is a given set of actions, and $\mu: \operatorname{Pr} \times \operatorname{Act} \times \mathcal{B}(\operatorname{Pr}) \rightarrow[0,1]$ is a so-called (general) transition probability function, i.e., $\mu(P, a, \cdot)$ is either the zero-map, or a Borel probability measure, for all $P \in \operatorname{Pr}, a \in$ Act.

A probabilistic bisimulation for a probabilistic transition system $(\operatorname{Pr}, A c t, \mu)$ is an equivalence ' $\equiv$ ' on $\operatorname{Pr}$ such that every equivalence class $E \subseteq \operatorname{Pr}$ of ' $\equiv$ ' is measurable, and

$$
P \equiv Q \Rightarrow \mu(P, a, E)=\mu(Q, a, E)
$$

for all $P, Q \in \operatorname{Pr}, a \in A c t$, and $E \in \operatorname{Pr} \equiv$. Two processes $P$ and $Q$ in $\operatorname{Pr}$ are said to be probabilistic bisimilar if there exists a probabilistic bisimulation containing the pair $(P, Q)$.

Note that the equivalence classes $E$ of ' $\equiv$ ' must be measurable, since only then the values $\mu(P, a, E)$ and $\mu(Q, a, E)$ are well-defined.

For reasons of presentation, we dispense, for a moment, with the actions and with the treatment of termination. They can be added again later. (Note that according to Definition 5.2, the Cartesian product $S \times T$ is admitted as a $\mathcal{M}_{1}$-bisimulation.) In this way, a probabilistic transition system becomes a function $\alpha: S \rightarrow \mathcal{M}_{1}(S)$ where $\mathcal{M}_{1}(S)$ denotes the collection of all Borel probability measures. In the reformulation of the related notion of probabilistic bisimulation we give, as before, first a slightly more general definition of bisimilarity of systems with different carriers.

Definition 5.2 Let $\alpha: S \rightarrow \mathcal{M}_{1}(S)$ and $\beta: T \rightarrow \mathcal{M}_{1}(T)$ be two probabilistic transition systems. A relation $R \subseteq S \times T$ is called a probabilistic bisimulation for $\alpha, \beta$ iff $R(s, t) \Rightarrow$ $\alpha(s)(U)=\beta(t)(V)$ for all $s \in S, t \in T$ and $U \in \mathcal{B}(S), V \in \mathcal{B}(T)$ such that $\pi_{1}^{-1}(U)=$ $\pi_{2}^{-1}(V)$. Two elements $s \in S, t \in T$ are said to be probabilistic bisimilar iff some probabilistic bisimulation contains the pair $(s, t)$. 
As for $\mathcal{D}$ in the previous section, $\mathcal{M}_{1}$ can be regarded as a functor, now on the category UMS of ultrametric spaces and nonexpansive mappings. For this purpose, $\mathcal{M}_{1}(M)$ should first of all be endowed with a metric structure. (For a similar result in the context of evaluations, see, e.g., [BK97].)

Lemma 5.3 Let $(M, d)$ be an ultrametric space. Put $\mathcal{O}_{\varepsilon}=\left\{O \in \mathcal{O} \mid \forall x \in O: B_{\varepsilon}(x) \subseteq O\right\}$, for $\varepsilon>0$. Let the distance $d$ on $\mathcal{M}_{1}(M)$, induced by $d$ on $M$, be given by

$$
d(\mu, \nu)=\inf \left\{\varepsilon>0 \mid \forall O \in \mathcal{O}_{\varepsilon}: \mu(O)=\nu(O)\right\} .
$$

Then $\left(\mathcal{M}_{1}(M), d\right)$ is an ultrametric space.

Proof Since $\mathcal{M}$ is an ultrametric space it holds that, for any $X \subseteq M, M \backslash\left(\bigcup_{x \in X} B_{\varepsilon}(x)\right)=$ $\bigcup_{x \in M \backslash X} B_{\varepsilon}(x)$, and $\mathcal{O}_{\varepsilon} \subseteq \mathcal{O}_{\varepsilon^{\prime}}$, for $\varepsilon \geq \varepsilon^{\prime}$. We check the various conditions of an ultrametric for the function $d$ on $\mathcal{M}_{1}(M)$.

The symmetry condition for $d$ is clear. Trivially, $d(\mu, \mu)=0$, for any $\mu \in \mathcal{M}_{1}(M)$. Now, suppose $d(\mu, \nu)=0$. By ultrametricity of $M, \mathcal{O}_{\varepsilon}$ is a $\sigma$-algebra: Clearly, $M \in \mathcal{O}_{\varepsilon}$; for $O \in \mathcal{O}_{\varepsilon}, O=\bigcup_{x \in O} B_{\varepsilon}(x)$, and it holds that $M \backslash O=M \backslash\left(\bigcup_{x \in O} B_{\varepsilon}(x)\right)=\bigcup_{x \in M \backslash O} B_{\varepsilon}(x)$, hence $M \backslash O \in \mathcal{O}_{\varepsilon}$; for $\left(O_{i}\right)_{i}$ in $\mathcal{O}_{\varepsilon}$ obviously $\bigcup_{i} O_{i} \in \mathcal{O}_{\varepsilon}$. The measures $\mu$ and $\nu$ coincide on the algebra $\bigcup\left\{\mathcal{O}_{\varepsilon} \mid \varepsilon>0\right\}$, and hence on the $\sigma$-algebra generated by it (see [Kec95, p106]). Any open set can be obtained as countable union of $O_{q}$ with $O_{q} \in \mathcal{O}_{q}, q \in \mathbb{Q}$. Hence, the $\sigma$-algebra generated by this collection is precisely the Borel $\sigma$-algebra of $M$. So, $\mu$ and $\nu$ coincide on $\mathcal{B}(M)$ and $\mu=\nu$.

The strong triangle inequality is verified as follows: Let $\mu, \nu, \rho \in \mathcal{M}_{1}(M)$ and pick $\varepsilon>0$ such that $\max \{d(\mu, \rho), d(\rho, \nu)\}<\varepsilon$. Then we have $d(\mu, \rho), d(\rho, \nu)<\varepsilon$, hence $\forall O \in \mathcal{O}_{\varepsilon}: \mu(O)=$ $\rho(O) \wedge \rho(O)=\nu(O)$. Thus $\forall O \in \mathcal{O}_{\varepsilon}: \mu(O)=\nu(O)$. So $d(\mu, \nu) \leq \varepsilon$. By taking the infimum over all $\varepsilon>0$ satisfying $\max \{d(\mu, \rho), d(\rho, \nu)\}<\varepsilon$ it follows that $d(\mu, \nu) \leq \max \{d(\mu, \rho), d(\rho, \nu)\}$.

Consider, as a concrete example, the set of strings $A=\{\epsilon, a, a b, a b c\}$ supplied with the Bairedistance, thus $d(\epsilon, w)=1$ for $w \in\{a, a b, a b c\}, d(a, w)=\frac{1}{2}$ for $w \in\{a b, a b c\}, d(a b, a b c)=\frac{1}{4}$. Let $\delta_{a}, \delta_{a b}$ be the Dirac measures for $a$ and $a b$, respectively. Since $d(a, a b)=\frac{1}{2}$ it follows that for any $\varepsilon>\frac{1}{2}$ and $O \in \mathcal{O}_{\varepsilon}, a \in O \Longleftrightarrow a b \in O$ and, consequently, $\delta_{a}(O)=\delta_{a b}(O)$ (viz. either

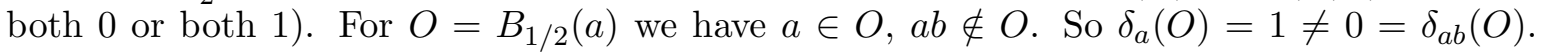
Therefore we have that $d\left(\delta_{a}, \delta_{a b}\right)=\inf \left\{\varepsilon \mid \varepsilon>\frac{1}{2}\right\}=\frac{1}{2}$.

Next we have to determine the action of $\mathcal{M}_{1}$ on nonexpansive functions.

Lemma 5.4 Let $M, N$ be two ultrametric spaces and let $f: M \rightarrow N$ be a nonexpansive mapping. Define, for $\mu \in \mathcal{M}_{1}(M)$, the function $\mathcal{M}_{1}(f)(\mu): \mathcal{B}(N) \rightarrow[0,1]$ by $\mathcal{M}_{1}(f)(\mu)(V)=$ $\mu\left(f^{-1}(V)\right)$. Then $\mathcal{M}_{1}(f): \mathcal{M}_{1}(M) \rightarrow \mathcal{M}_{1}(N)$ is well-defined and nonexpansive.

Proof The well-definedness of $\mathcal{M}_{1}(f)$, i.e. the fact that $\mathcal{M}_{1}(f)(\mu)$ is a Borel probability measure on $N$ for any $\mu \in \mathcal{M}_{1}(M)$ is standard. The nonexpansiveness of $\mathcal{M}_{1}(f)$ is based on the fact that $O \in \mathcal{O}_{\varepsilon}(N) \Rightarrow f^{-1}(O) \in \mathcal{O}_{\varepsilon}(M)$, for any $\varepsilon>0$ : For, suppose $O \in \mathcal{O}_{\varepsilon}(N)$ and $x, y \in M$ such that $x \in f^{-1}(O)$ and $d(x, y)<\varepsilon$, then $f(x) \in O$ and $d(f(x), f(y))<\varepsilon$ by nonexpansiveness of $f$. Since $O \in \mathcal{O}_{\varepsilon}(N)$ it follows that $f(y) \in O$ and, consequently, $y \in f^{-1}(O)$. Now, let $\mu, \nu \in \mathcal{M}_{1}(M)$. Suppose $d(\mu, \nu)<\varepsilon$ for some $\varepsilon>0$, i.e. $\forall O \in \mathcal{O}_{\varepsilon}(M): \mu(O)=\nu(O)$. Pick $O \in \mathcal{O}_{\varepsilon}(N)$. Note that $f^{-1}(O) \in \mathcal{O}_{\varepsilon}(M)$. So, by definition of $\mathcal{M}_{1}(f)(\mu)$ and $\mathcal{M}_{1}(f)(\nu)$, 
$\mathcal{M}_{1}(f)(\mu)(O)=\mu\left(f^{-1}(O)\right)=\nu\left(f^{-1}(O)\right)=\mathcal{M}_{1}(f)(\nu)(O)$. Taking the infimum over $\varepsilon$ yields that $d\left(\mathcal{M}_{1}(f)(\mu), \mathcal{M}_{1}(f)(\nu)\right) \leq d(\mu, \nu)$, and it follows that $\mathcal{M}_{1}(f): \mathcal{M}_{1}(M) \rightarrow \mathcal{M}_{1}(N)$ is a nonexpansive mapping.

As an aside, $\mathcal{M}_{1}(f)$ would not be nonexpansive when $\mathcal{M}_{1}(M)$ and $\mathcal{M}_{1}(N)$ are supplied with the distance of uniform convergence $d_{F}$ inherited from the function spaces $\mathcal{B}(M) \rightarrow[0,1]$ and $\mathcal{B}(N) \rightarrow[0,1]$. Then we would have, for $\mu, \nu \in \mathcal{M}_{1}(M)$, by definition of the distance $d_{F}$,

$$
d_{F}(\mu, \nu)=\sup \{|\mu(U)-\nu(U)| \mid U \in \mathcal{B}(M)\} .
$$

If we then consider, for example, the identity mapping id on the set $A=\{\epsilon, a, a b, a b c\}$ mentioned above, we have, for the Borel set $\{a\}$,

$$
\mathcal{M}_{1}(\mathrm{id})\left(\delta_{a}\right)(\{a\})=\delta_{a}(\{a\})=1 \text { and } \mathcal{M}_{1}(\mathrm{id})\left(\delta_{a b}\right)(\{a\})=\delta_{a b}(\{a\})=0 .
$$

So $d_{F}\left(\mathcal{M}_{1}(\mathrm{id})\left(\delta_{a}\right), \mathcal{M}_{1}(\mathrm{id})\left(\delta_{a b}\right)\right)=1$ and, with respect to the metrics involved here, $\mathcal{M}_{1}(\mathrm{id})$ would not be nonexpansive. However, as Lemma 5.4 shows, with respect to the distance introduced in Lemma 5.3, $\mathcal{M}_{1}($ id $)$ is nonexpansive. As a remark, the construction does not apply to the metric, but not ultrametric, space $\mathbb{R}$ endowed with the Euclidean distance, which can possibly complicate the exploitation of the theory developed here in concrete applications.

By the previous lemmas we can view $\mathcal{M}_{1}$ as a functor on UMS, the category of ultrametric spaces with nonexpansive mappings. Following the coalgebraic paradigm, $\mathcal{M}_{1}$ induces a notion of $\mathcal{M}_{1}$-bisimulation. One half of the relationship of $\mathcal{M}_{1}$-bisimulation and probabilistic bisimulation can be shown directly.

Lemma 5.5 Let $\alpha: S \rightarrow \mathcal{M}_{1}(S), \beta: T \rightarrow \mathcal{M}_{1}(T)$ be two probabilistic transition systems. Any $\mathcal{M}_{1}$-bisimulation $R$ for $\alpha$ and $\beta$ is also a probabilistic bisimulation for $\alpha, \beta$.

Proof Similar to the proof of Lemma 4.3.

Below we show that the reverse of Lemma 5.5 holds, under reasonable conditions. The technicality to be dealt with concerns the proper generalization of the measurability condition of the equivalence classes $E$.

For a probabilistic bisimulation ' $\equiv$ ' in the sense of Definition 5.1 we have a partitioning of the carrier into equivalence classes and of ' $\equiv$ ' into squares of equivalence classes. Moreover, these subsets are measurable by assumption. So, we have $\equiv=\bigcup_{i \in I} E_{i} \times E_{i}$ with each $E_{i}$ measurable. Similarly, for the general set-up, we want a decomposition $R=\bigcup_{k \in K} E_{k} \times F_{k}$ where the $E_{k}$ and $F_{k}$ are Borel sets in $S$ and $T$, respectively. Additionally, for measure theoretical considerations, we will assume the number of rectangles $E_{k} \times F_{k}$ that constitute $R$ to be countable.

Definition 5.6 A binary relation $R \subseteq S \times T$ on two ultrametric spaces $S$ and $T$ is said to have a Borel decomposition iff $R=\bigcup_{k \in K} E_{k} \times F_{k}$ where $\left\{E_{k} \mid k \in K\right\},\left\{F_{k} \mid k \in K\right\}$ are countable partitions of Borel sets of $S$ and $T$, respectively.

Discrete probabilistic bisimulations are examples of relations that are Borel decomposable. If $\alpha$ is a simple probability distribution over $s_{1}, \ldots, s_{n}$ and $\beta$ over $t_{1}, \ldots, t_{m}$ then $R$ can be partitioned into Cartesian products of finite sets and the (measurable) rectangle $S \backslash\left\{s_{1}, \ldots, s_{n}\right\} \times$ $T \backslash\left\{t_{1}, \ldots, t_{m}\right\}$. Note that for $R$ z-closed, we have in fact $R=\bigcup\{E(t) \times F(s) \mid(s, t) \in R\}$ but, in general, $E(t) \times F(s)$ need not be measurable. 
In the construction of a mediating probabilistic transition system $\gamma: R \rightarrow \mathcal{M}_{1}(R)$, for a given probabilistic bisimulation $R$, we can again assume that $R$ is z-closed. Since no measure theoretical considerations are involved, the proof of this is literally as for Lemma 4.4.

Lemma 5.7 If $R \subseteq S \times T$ is a probabilistic bisimulation for $\alpha: S \rightarrow \mathcal{M}_{1}(S), \beta: T \rightarrow \mathcal{M}_{1}(T)$, then so is $R^{*}$, the $z$-closure of $R$.

We now have arrived at the main result. Using notations of Section 2, we have

$$
\pi_{1}^{-1}(U)=(U \times F[U]) \cap R \text { and } \pi_{2}^{-1}(V)=(V \times E[V]) \cap R .
$$

Moreover, if $R$ has a Borel decomposition $R=\bigcup_{k \in K} E_{k} \times F_{k}$, it holds that

$$
F[U] \cap F_{k}=F_{k} \text { if } U \cap E_{k} \neq \emptyset \text {, and } E[V] \cap E_{k}=E_{k} \text { if } V \cap F_{k} \neq \emptyset .
$$

These facts will be employed in the calculations for the proof of the next theorem.

Theorem 5.8 Let $\alpha: S \rightarrow \mathcal{M}_{1}(S), \beta: T \rightarrow \mathcal{M}_{1}(T)$ be two probabilistic transition systems. Let $R$ be a probabilistic bisimulation for $\alpha, \beta$ in the sense of Definition 5.2. Assume that $R$ is z-closed. If $R$ has a Borel decomposition, then $R$ is an $\mathcal{M}_{1}$-bisimulation for $\alpha, \beta$.

Proof Let $\left\{E_{k} \times F_{k} \mid k \in K\right\}$ be a Borel decomposition of $R$. Suppose $R(s, t)$ holds. Since $R$ is a probabilistic bisimulation for $\alpha, \beta$ we have $\alpha(s)\left(E_{k}\right)=\beta(t)\left(F_{k}\right)$. In particular,

$$
\alpha(s)\left(E_{k}\right)=0 \Longleftrightarrow \beta(t)\left(F_{k}\right)=0,
$$

for all $k \in K$. Let $K^{\prime} \subseteq K$ be the subset of indices for which $\beta(t)\left(F_{k}\right)$ is nonzero. Let $\gamma_{s, t}$ be short for $\gamma(s, t)$, and put

$$
\gamma_{s, t}((U \times V) \cap R)=\sum_{k \in K^{\prime}} \frac{\alpha(s)\left(U \cap E_{k}\right) \cdot \beta(t)\left(V \cap F_{k}\right)}{\beta(t)\left(F_{k}\right)}
$$

for $U \in \mathcal{B}(S), V \in \mathcal{B}(T)$. Note that the denominators occurring in equation (5.5) do not depend on the sets $U$ and $V$. We check that $\gamma_{s, t}$ is a Borel probability measure. One readily verifies that $\gamma_{s, t}$ is $\sigma$-additive. The latter follows from the $\sigma$-additivity of the product measure $\alpha(s) \times \beta(t)$ and the observation that disjointness on $R$ can be propagated to disjointness on $S \times T$. Moreover, by a straightforward calculation relying on the totality-assumption $\bigcup_{k \in K} E_{k}=S, \gamma_{s, t}(R)=\gamma_{s, t}((S \times T) \cap R)=1$. It follows that equation (5.5) uniquely determines a Borel measure on $R$.

We claim that $\gamma_{s, t}$ is mediating for $\alpha(s)$ and $\beta(t)$ : On the one hand we have, for $U \in \mathcal{B}(S)$,

$$
\begin{aligned}
& \mathcal{M}_{1}\left(\pi_{1}\right)\left(\gamma_{s, t}\right)(U) \\
& =\left[\text { def. } \mathcal{M}_{1} \text {, eq. (5.2)] } \gamma_{s, t}((U \times F[U]) \cap R)\right. \\
& =[\text { def. } \gamma] \quad \sum_{k \in K^{\prime}} \frac{\alpha(s)\left(U \cap E_{k}\right) \cdot \beta(t)\left(F[U] \cap F_{k}\right)}{\beta(t)\left(F_{k}\right)} \\
& =\left[\text { eq. (5.3)] } \sum_{k \in K} \alpha(s)\left(U \cap E_{k}\right)\right. \\
& =\left[\sigma \text {-add., } \bigcup_{k} E_{k} \text { partition of } S\right] \alpha(s)(U),
\end{aligned}
$$


and, on the other hand, for $V \in \mathcal{B}(T)$,

$$
\begin{aligned}
& \gamma_{s, t}\left(\pi_{2}^{-1}(V)\right) \\
& \quad=[\text { eq. (5.2), def. } \gamma] \sum_{k \in K^{\prime}} \frac{\alpha(s)\left(E[V] \cap E_{k}\right) \cdot \beta(t)\left(V \cap F_{k}\right)}{\beta(t)\left(F_{k}\right)} \\
& \quad=[\text { eq. (5.3), } \beta(t) \text { strict }] \sum_{k \in K^{\prime}} \frac{\alpha(s)\left(E_{k}\right) \cdot \beta(t)\left(V \cap F_{k}\right)}{\beta(t)\left(F_{k}\right)} \\
& \stackrel{(*)}{=}[R \text { prob. bisim. }] \quad \sum_{k \in K^{\prime}} \beta(t)\left(V \cap F_{k}\right) \\
& \quad=[\text { as before }] \beta(t)(V) .
\end{aligned}
$$

At $(*)$ we have used that $R$ is a probabilistic bisimulation and the observation that $\pi_{1}^{-1}\left(E_{k}\right)=$ $E_{k} \times F_{k}=\pi_{2}^{-1}\left(F_{k}\right)$. Since the rectangles $U \times V$ generate $\gamma_{s, t}$, it follows that $\mathcal{M}_{1}\left(\pi_{1}\right)\left(\gamma_{s, t}\right)=$ $\alpha(s)=\alpha\left(\pi_{1}(s, t)\right)$ and, by symmetry, that $\mathcal{M}_{1}\left(\pi_{2}\right)\left(\gamma_{s, t}\right)=\beta(t)=\beta\left(\pi_{2}(s, t)\right)$.

In the remainder of this section, we shall again use some general insights from the theory of coalgebras, this time by applying a result from [AR89, RT93]. By currying, a probabilistic transition system $(\operatorname{Pr}, A c t, \mu)$ can be interpreted as a map $\mu: \operatorname{Pr} \rightarrow\left(A c t \rightarrow\left(\mathcal{M}_{1}(\operatorname{Pr})+\{\mathbf{0}\}\right)\right)$. The special element $\mathbf{0}$ corresponds to the zero-map (that is allowed by Definition 5.1). So, from the coalgebraic point of view, probabilistic transition systems are coalgebras of the functor $\operatorname{Act} \rightarrow\left(\mathcal{M}_{1}(\cdot)+\{\mathbf{0}\}\right)$.

In turns out that we are only able to show the existence of a final coalgebra when we consider an adaptation of $\mathcal{M}_{1}$, say $\mathcal{M}_{1}^{\prime}$, which delivers Borel probability measures with socalled compact support, i.e., measures that vanish outside a compact set. More precisely, for a metric space $M, \mu: \mathcal{B}(M) \rightarrow[0,1]$ is said to have a compact support if there exists a compact subset $K \subseteq M$ such that $U \cap K=\emptyset \Rightarrow \mu(U)=0$, for all $U \in \mathcal{B}(M)$. Let $\mathcal{M}_{1}^{\prime}(M)$ denote the collection of all Borel probability measures of an ultrametric space $M$ with a compact support. Similarly as for $\mathcal{M}_{1}$, the new $\mathcal{M}_{1}^{\prime}$ extends to a functor on UMS.

Lemma 5.9 Let the functor $\mathcal{M}_{1}^{\prime}$ on UMS be given as follows:

$$
\begin{aligned}
\mathcal{M}_{1}^{\prime}(M) & =\left\{\mu \in \mathcal{M}_{1}(M) \mid \mu \text { has compact support }\right\} \\
\mathcal{M}_{1}^{\prime}(f) & =\lambda \mu \cdot \lambda V \cdot \mu\left(f^{-1}(V)\right)
\end{aligned}
$$

for ultrametric spaces $M, N$ and $f: M \rightarrow N$ nonexpansive. Then $\mathcal{M}_{1}^{\prime}$ is well-defined.

Proof The only thing to check is that $\mathcal{M}_{1}^{\prime}(f)(\mu)$ has a compact support for $f: M \rightarrow N$ nonexpansive and $\mu \in \mathcal{M}_{1}^{\prime}(M)$. So suppose $\mu \in \mathcal{M}_{1}^{\prime}(M)$ and $K \subseteq M$ is compact with the property $U \cap K=\emptyset \Rightarrow \mu(U)=0$, for all $U \in \mathcal{B}(M)$. Since $f$ is continuous, the direct image $f[K]$ is compact in $N$. Note that $\mathcal{M}_{1}^{\prime}(f)(\mu)$ vanishes outside $f[K]$, i.e. $V \cap f[K]=\emptyset \Rightarrow$ $\mathcal{M}_{1}^{\prime}(f)(\mu)(V)=0$, since, for $V \in \mathcal{B}(N)$ such that $V \cap f[K]=\emptyset$, we have $\mathcal{M}_{1}^{\prime}(f)(\mu)(V)=$ $\mu\left(f^{-1}(V)\right)=0$ as $V \cap f[K]=\emptyset$ implies $f^{-1}(V) \cap K=\emptyset$. 
The functor $\mathcal{M}_{1}^{\prime}$ is the key building block of the functor $\mathcal{F}$ below that captures general probabilistic transition systems. Lemma 5.10 collects the relevant properties of $\mathcal{M}_{1}^{\prime}$. Some preparatory metric definitions and facts are presented first.

For two subsets $X, Y \subseteq M$ of an ultrametric space $M$, the Hausdorff-distance $d_{H}(X, Y)$ is given by

$$
d_{H}(X, Y)=\inf \left\{\varepsilon \mid \forall O \in \mathcal{O}_{\varepsilon}: O \cap X=\emptyset \Longleftrightarrow O \cap Y=\emptyset\right\} .
$$

We will employ the following result: If $\mathcal{M}$ is a complete ultrametric space, then the collection $\mathcal{P}_{c o}(M)$ of all compact subsets of $M$ supplied with the Hausdorff-distance is also a complete metric space. (For a proof of this fact the reader may consult, e.g., [BV96], Appendix A.).

For a Borel probability measure $\mu$ with compact support we define its support $\operatorname{spt}(\mu)$ by

$$
\operatorname{spt}(\mu)=\bigcap\{K \subseteq M \mid K \text { compact, } \mu \text { vanishes outside } K\} .
$$

For any open $O \in \mathcal{O}(M)$ we then have the equivalence $\mu(O)=0 \Longleftrightarrow \operatorname{spt}(\mu) \cap O=\emptyset$. (See, e.g., [Rud66, p57].)

\section{Lemma 5.10}

(a) If $M$ is a complete ultrametric space, then $\mathcal{M}_{1}^{\prime}(M)$ is complete too.

(b) The functor $\mathcal{M}_{1}^{\prime}$ on UMS is locally nonexpansive, i.e., for all ultrametric spaces $M, N$ the mapping $\left(M \rightarrow_{1} N\right) \rightarrow\left(\mathcal{M}_{1}^{\prime}(M) \rightarrow_{1} \mathcal{M}_{1}^{\prime}(N)\right)$ such that $f \mapsto \mathcal{M}_{1}^{\prime}(f)$ is nonexpansive with respect to the distance $d_{F}$ of uniform convergence.

\section{Proof}

(a) Suppose $\left(\mu_{i}\right)_{i}$ is a Cauchy-sequence in $\mathcal{M}_{1}^{\prime}(M)$. Put $\mathcal{O}^{\prime}=\bigcup\left\{\mathcal{O}_{\varepsilon} \mid \varepsilon>0\right\}$. For $O \in \mathcal{O}_{\varepsilon}$, the sequence $\left(\mu_{i}(O)\right)_{i}$ is eventually constant. Define $\mu: \mathcal{O}^{\prime} \rightarrow[0,1]$ by $\mu(O)=\lim _{i} \mu_{i}(O)$.

We claim that $\mu$ vanishes outside a compactum $K \subseteq M$ : Put $K_{i}=\operatorname{spt}\left(\mu_{i}\right)$. If $d\left(\mu_{i}, \mu_{j}\right)<$ $\varepsilon$, then $\mu_{i}\left(B_{\varepsilon}(x)\right)=0 \Longleftrightarrow \mu_{j}\left(B_{\varepsilon}(x)\right)=0$, so $B_{\varepsilon}(x) \cap K_{i}=\emptyset \Longleftrightarrow B_{\varepsilon}(x) \cap K_{j}=\emptyset$, for any $x \in M$. Hence $d_{H}\left(K_{i}, K_{j}\right) \leq \varepsilon$. It follows that $\left(K_{i}\right)_{i}$ is a Cauchy sequence in $\mathcal{P}_{c o}(M)$. Put $K=\lim _{i} K_{i}$. For $O \in \mathcal{O}_{\varepsilon}$ and $i$ such that $j \geq i$ implies $d_{H}\left(K_{j}, K\right)<\varepsilon$ and $d\left(\mu_{j}, \mu\right)<\varepsilon$ we have $O \cap K=\emptyset \Longleftrightarrow \forall j \geq i: O \cap K_{j}=\emptyset \Longleftrightarrow \forall j \geq i: \mu_{j}(O)=$ $0 \Longleftrightarrow \mu(O)=0$.

Next we verify that $\mu$ is $\sigma$-additive. Clearly, $\mu$ is finitely additive, since all $\mu_{i}$ are. Suppose $\left(O_{i}\right)_{i}$ is a disjoint sequence in $\mathcal{O}^{\prime}$ such that $\bigcup_{i} O_{i} \in \mathcal{O}^{\prime}$. Put $O^{\prime}=M \backslash\left(\bigcup_{i} O_{i}\right)$. By ultrametricity $O^{\prime}$ is open. Now $\left\{O_{i} \mid i \in \mathbb{N}\right\} \cup\left\{O^{\prime}\right\}$ is a disjoint cover of $K$. Thus the index set $I$ such that $I=\left\{i \mid O_{i} \cap K \neq \emptyset\right\}$ is finite. Therefore,

$$
\begin{aligned}
& \sum_{i} \mu\left(O_{i}\right) \\
& =\left[\mu\left(O_{i}\right)=0 \text { for } i \notin I\right] \quad \sum_{i \in I} \mu\left(O_{i}\right) \\
& =[\text { finite additivity }] \mu\left(\bigcup_{i \in I} \mu\left(O_{i}\right)\right) \\
& =\left[\left(\bigcup_{i \notin I} O_{i}\right) \cap K=\emptyset\right] \quad \mu\left(\bigcup_{i \in i} O_{i}\right)+\mu\left(\bigcup_{i \notin I} O_{i}\right) \\
& =[\text { finite additivity }] \mu\left(\bigcup_{i} O_{i}\right) .
\end{aligned}
$$


Now, by general measure theoretic considerations, $\mu$ extends uniquely to a Borel probability measure of with support $K$.

(b) Let $f, g: M \rightarrow N$ be nonexpansive. To show, $d_{F}\left(\mathcal{M}_{1}^{\prime}(f), \mathcal{M}_{1}^{\prime}(g)\right) \leq d_{F}(f, g)$. Suppose, $d_{F}(f, g)<\varepsilon, \mu \in \mathcal{M}_{1}^{\prime}(M)$ and $O \in \mathcal{O}_{\varepsilon}(N)$. Note, $f^{-1}(O)=g^{-1}(O)$. Therefore $\mathcal{M}_{1}^{\prime}(f)(\mu)(O)=\mu\left(f^{-1}(O)\right)=\mu\left(g^{-1}(O)\right)=\mathcal{M}_{1}^{\prime}(g)(\mu)(O)$. Consequently we have that $d\left(\mathcal{M}_{1}^{\prime}(f)(\mu), \mathcal{M}_{1}^{\prime}(g)(\mu)\right) \leq \varepsilon$ in $\mathcal{M}_{1}^{\prime}(N)$ and $d_{F}\left(\mathcal{M}_{1}^{\prime}(f), \mathcal{M}_{1}^{\prime}(g)\right) \leq d_{F}(f, g)$ follows.

Lemma 5.10 paves the way of using the functor $\mathcal{M}_{1}^{\prime}$ in our categorical set-up. It is the main ingredient in the functor $A c t \rightarrow\left(\mathcal{M}_{1}^{\prime}(\cdot) / 2+\{\mathbf{0}\}\right)$ on UMS. The coalgebras of this functor are general probabilistic transition systems. To ensure the technical property of local contractiveness (see, e.g., [RT93]), we have put in the scaling functor $\cdot / 2$. The functor $\cdot / 2$ : UMS $\rightarrow$ UMS maps a space $M$ to the space with the same underlying set but now with all distances multiplied by a factor $\frac{1}{2}$. This operation is harmless from a semantical point of view. The usage of $\mathcal{M}_{1}^{\prime}$, though, does narrow the type of transition systems falling within the framework. However, we stress that the established relationship of coalgebraic and probabilistic bisimulation, still carries through for the modified setting. Additionally, for the class of transition systems, now captured by the functor $A$ ct $\rightarrow\left(\mathcal{M}_{1}^{\prime}(\cdot) / 2+\{\mathbf{0}\}\right)$, the existence of a final coalgebra is guaranteed.

Theorem 5.11 Let the functor $\mathcal{F}: \mathbf{U M S} \rightarrow$ UMS be given by $\mathcal{F}=$ Act $\rightarrow\left(\mathcal{M}_{1}^{\prime}(\cdot) / 2+\{\mathbf{0}\}\right)$. Then the following holds:

(a) $\mathcal{F}$ is locally contractive, i.e., for some $\kappa, 0 \leq \kappa<1$, and all ultrametric spaces $M$ and $N$, the function $\mathcal{F}_{M, N}:\left(M \rightarrow{ }_{1} N\right) \rightarrow\left(\mathcal{F}(M) \rightarrow{ }_{1} \mathcal{F}(N)\right)$ given by $\mathcal{F}_{M, N}(f)=\mathcal{F}(f)$ is $\kappa$-contractive.

(b) If $M$ is complete, then $\mathcal{F}(M)$ is complete.

(c) The functor $\mathcal{F}$ has a final coalgebra.

Proof The presence of ' $\cdot / 2$ ' in the definition of $\mathcal{F}$ results in (a). The other constituent functors are locally nonexpansive. Only for part (b) the assumption of measures having a compact support is necessary. Part (b) is an immediate consequence of Lemma 5.10. Finally, part (c) follows from (a) and (b), and (a minor variation of) [RT93, Thm 4.8].

Let $\mathbb{Q}$ be the final $\mathcal{F}$-coalgebra: $\mathbb{Q} \cong A c t \rightarrow\left(\mathcal{M}_{1}^{\prime}(\mathbb{Q}) / 2+\{\mathbf{0}\}\right)$. From Theorem 3.1 and 5.11 we then immediately obtain the following result.

Corollary 5.12 The system $\mathbb{Q}$ is internally fully abstract with respect to probabilistic bisimulation.

Next we would like to apply Theorem 3.2 to generalize the above internal full abstractness result, similar to Theorem 4.8 for the discrete case. We do not know, however, whether $\mathcal{F}$ preserves weak pullbacks. This is to be investigated further, notably in view of the results of [BDEP97, DEP98], which seem to be closely related to this question. 


\section{Conclusion and future research}

In this paper, a framework is proposed for probabilistic transition systems, involving general probability measures, and an associated notion of probabilistic bisimulation. Most research reported in the literature so far deals with discrete probabilistic transition systems, employing simple probability distributions only. The use of Borel measures allows for an extension of this to a setting for which discreteness and image-finiteness are too restrictive.

Following the transition-systems-as-coalgebras paradigm, the categorical set-up provides a characterization of the Larsen-Skou bisimulation in terms of a set functor. For the continuous case, a similar result is shown for a functor on the category of ultrametric spaces. Moreover, exploiting parts of the theory of coalgebras, both for the discrete case and for the continuous case, internally fully abstract domains are constructed.

Further investigations of the proposed notion of Borel decomposition should clarify how the latter relates to the use of Polish spaces as in [BDEP97]. We expect that the technical result obtained there, on the existence of weak pullbacks, applies also to our setting. Other related work includes [BK97] where discrete probabilistic bisimulation is discussed in the context of CCS extended with probabilistic choice, and [Mos97] where coalgebraic logics also based on functors, including our functor $\mathcal{D}$, are studied. Connections with modal logics are considered by Larsen and Skou and by Desharnais et al. as well. Another direction for future research concerns the usage of the domains obtained as final coalgebra of a functor involving $\mathcal{M}_{1}^{\prime}$. Once a suitable continuous process language is identified (such as PCCS [GJS90] for the discrete case), the domain $\mathbb{Q}$ of Section 5 (or a variation thereof) may serve a promising candidate for its modeling. We expect that a nonuniform extension of the CSP-style language treated by Seidel [Sei95] can be interpreted in this model. Also, the relationship with other work of the research group at Oxford, in particular on weakest precondition semantics, can now be studied using the dualities as studied in [Bon96]. Finally, we hope that the process equivalences and fully abstract domains proposed in this paper may contribute to the semantical study of dynamical and hybrid systems.

\section{References}

[Acz88] P. Aczel. Non-Well-Founded Sets. CSLI Lecture Notes 14. Center for the Study of Languages and Information, Stanford, 1988.

[AM89] P. Aczel and N. Mendler. A final coalgebra theorem. In D.H. Pitt, D.E. Rydeheard, P. Dybjer, A.M. Pitts, and A. Poigne, editors, Proc. Category Theory and Computer Science, pages 357-365. Lecture Notes in Computer Science 389, 1989.

[AR89] P. America and J.J.M.M. Rutten. Solving reflexive domain equations in a category of complete metric spaces. Journal of Computer Systems and Sciences, 39:343-375, 1989.

[Bar93] M. Barr. Terminal coalgebras in well-founded set theory. Theoretical Computer Science, 114:299-315, 1993. See also the addendum, Theoretical Computer Science, 124:189-192, 1994.

[BDEP97] R. Blute, J. Desharnais, A. Edalat, and P. Panangaden. Bisimulation for labeled Markov processes. In Proc. Twelfth Annual IEEE Symposium On Logic In Com- 
puter Science, pages 149-159, Warsaw, 1997. Full version submitted to Information and Computation.

[BG96] M. Bernardo and R. Gorrieri. Extended Markovian process algebra. In U. Montanari and V. Sassone, editors, Proc. CONCUR'96, pages 315-330. Lecture Notes in Computer Science 1119, 1996.

[BK97] C. Baier and M. Kwiatkowska. Domain equations for probabilistic processes. Technical Report CSR-97-7, University of Birmingham, 1997. Also appeared in Proc. EXPRESS'97, Electronic Notes in Theoretical Computer Science, volume 7, Elsevier Science 1997.

[BLFG95] A. Benveniste, B.C. Levy, E. Fabre, and Paul Le Guernic. A calculus of stochastic systems for the specification, simulation, and hidden state estimation of mixed stochastic/nonstochastic systems. Theoretical Computer Science, 152:171-217, 1995.

[Bol79] B. Bollobás. Graph theory, volume 63 of Graduate Texts in Mathematics. SpringerVerlag, 1979.

[Bon96] M.M. Bonsangue. Topological Dualities in Semantics. PhD thesis, Vrije Universiteit Amsterdam, 1996. A revised version appeared as Electronic Notes in Theoretical Computer Science, volume 8, Elsevier Science 1997.

[BV96] J.W. de Bakker and E.P. de Vink. Control Flow Semantics. The MIT Press, 1996.

[DEP98] J. Desharnais, A. Edalat, and P. Panangaden. A logical characterization of bisimulation for labeled Markov processes. In Proc. Thirteenth Annual IEEE Symposium On Logic In Computer Science, pages 478-487, Indianapolis, 1998.

[Eda95a] A. Edalat. Domain theory and integration. Theoretical Computer Science, 151:163-193, 1995.

[Eda95b] A. Edalat. Dynamical systems, measures and fractals via domain theory. Information and Computation, 120:32-48, 1995.

[Gir81] M. Giry. A categorical approach to probability theory. In B. Banaschewski, editor, Proc. Categorical Aspects of Topology and Analysis, pages 68-85. Lecture Notes in Mathematics 915, 1981.

[GJS90] A. Giacalone, C. Jou, and S.A. Smolka. Algebraic reasoning for probabilistic concurrent systems. In M. Broy and C.B Jones, editors, Proc. Working Conference on Programming Concepts and Methods. IFIP TC2, Sea of Gallilee, 1990.

[GSS95] R.J. van Glabbeek, S.A. Smolka, and B. Steffen. Reactive, generative and stratified models of probabilistic processes. Information and Computation, 121:59-80, 1995. Preliminary version (co-authored by C.M.N. Tofts) appeared in Proc. LICS'90, Philadelphia, 1990.

[Hen95] T.A. Henzinger. Hybrid automata with finite bisimulations. In Z. Fülöp and F. Gécseg, editors, Proc. ICALP'95, pages 324-335. Lecture Notes in Computer Science 944, 1995. 
[Jac96] B. Jacobs. Objects and classes, co-algebraically. In B. Freitag, C.B. Jones, C. Lengauer, and H.-J. Schek, editors, Object-Orientation with Parallelism and Persistence. Kluwer, 1996.

[JL91] B. Jonsson and K.G. Larsen. Specification and refinement of probabilistic processes. In Proc. LICS'91, pages 266-277. Amsterdam, 1991.

[JNW96] A. Joyal, M. Nielsen, and G. Winskel. Bisimulation from open maps. Information and Computation, 127:164-185, 1996.

[Jon89] C. Jones. Probabilistic Non-determinism. PhD thesis, University of Edinburgh, 1989.

[JP89] C. Jones and G. Plotkin. A probabilistic powerdomain of evaluations. In Proc. LICS'89, pages 186-195. Asilomar, 1989.

[JR97] B. Jacobs and J.J.M.M. Rutten. A tutorial on (co)algebras and (co)induction. Bulletin of EATCS, 62:222-259, 1997.

[Kec95] A.S. Kechris. Classical Descriptive Set Theory, volume 156 of Graduate Texts in Mathematics. Springer, 1995.

[Kel76] R.M. Keller. Formal verification of parallel programs. Communications of the ACM, 19:371-384, 1976.

[KN96] M. Kwiatkowska and G. Norman. Probabilistic metric semantics for a simple language with recursion. In W. Penczek and A. Szalas, editors, Proc. MFCS'96, pages 419-430. Lecture Notes in Computer Science 1113, 1996.

[KV84] K. Kunen and J.E. Vaughan, editors. Handbook of Set-Theoretic Topology. NorthHolland, 1984.

[LS91] K.G. Larsen and A. Skou. Bisimulation through probabilistic testing. Information and Computation, 94:1-28, 1991.

[MG85] J. Meseguer and J.A. Goguen. Initiality, induction, and computability. In M. Nivat and J.C. Reynolds, editors, Algebraic Methods in Semantics, pages 459-541. Cambridge University Press, 1985.

[Mil80] R. Milner. A Calculus of Communicating Systems. Lecture Notes in Computer Science 92, 1980.

[Mos97] L.S. Moss. Coalgebraic logic. Draft, 1997. To appear in Annals of Pure and Applied Logic.

[Par81] D.M.R. Park. Concurrency and automata on infinite sequences. In P. Deussen, editor, Proc. 5th GI Conference on Theoretical Computer Science, pages 167-183. Lecture Notes in Computer Science 104, 1981.

[Plo81] G.D. Plotkin. A structural approach to operational semantics. Report DAIMI FN-19, Aarhus University, 1981. 
[Rei95] H. Reichel. An approach to object semantics based on terminal coalgebras. Mathematical Structures in Computer Science, 5:129-152, 1995.

[RT93] J.J.M.M. Rutten and D. Turi. On the foundations of final semantics: non-standard sets, metric spaces, partial orders. In J.W. de Bakker, W.-P. de Roever, and G. Rozenberg, editors, Proc. REX Workshop on Semantics: Foundations and Applications, pages 477-530. Lecture Notes in Computer Science 666, 1993.

[RT94] J.J.M.M. Rutten and D. Turi. Initial algebra and final coalgebra semantics for concurrency. In J.W. de Bakker, W.-P. de Roever, and G. Rozenberg, editors, Proc. REX School/Symposium 'A Decade of Concurrency', pages 530-582. Lecture Notes in Computer Science 803, 1994.

[Rud66] W. Rudin. Real and Complex Analysis. McGraw-Hill, 1966.

[Rut96] J.J.M.M. Rutten. Universal coalgebra: a theory of systems. Report CS-R9652, CWI, 1996. Ftp-available at ftp.cwi.nl as pub/CWIreports/AP/CS-R9652.ps.Z. To appear in Theoretical Computer Science.

[Seg95] R. Segala. Modeling and Verification of Randomized Distributed Real-Time Systems. PhD thesis, MIT, 1995.

[Sei95] K. Seidel. Probabilistic communicating processes. Theoretical Computer Science, 152:219-249, 1995.

\section{Appendix}

We shall discuss (weak) pullbacks in the category Set of sets and functions, and prove that the functor $\mathcal{D}:$ Set $\rightarrow$ Set, defined on a set $S$ by

$$
\mathcal{D}(S)=\{\mu: S \rightarrow[0,1] \mid \mu \text { is a simple probability distribution }\}
$$

and on a function $f: S \rightarrow T$ by $\mathcal{D}(f)(\mu)(t)=\mu\left[f^{-1}(\{t\})\right]$, for $\mu$ in $\mathcal{D}(S)$ and $t$ in $T$, preserves weak pullbacks.

A pullback of functions $f: S \rightarrow T$ and $g: U \rightarrow T$ is a triple $(P, k: P \rightarrow S, l: P \rightarrow U)$ with $f \circ k=g \circ l$ such that for any set $X$ and functions $i: X \rightarrow S$ and $j: X \rightarrow U$ with $f \circ i=g \circ j$ there exists a unique (so-called mediating) function $h: X \rightarrow P$ with $k \circ h=i$ and $l \circ h=j$. In Set, a pullback of functions $f: S \rightarrow T$ and $g: U \rightarrow T$ always exists: the set

$$
P=\{\langle s, u\rangle \in S \times U \mid f(s)=g(u)\},
$$

with projections $\pi_{1}: P \rightarrow S$ and $\pi_{2}: P \rightarrow U$, is a pullback of $f$ and $g$.

A weak pullback is defined in the same way as a pullback, but without the requirement that the mediating function be unique. Equivalently, for any $s$ in $S$ and $u$ in $U$ such that $f(s)=g(u)$ there exists a (not necessarily unique) element $p$ in $P$ with $k(p)=s$ and $l(p)=$ $u$. A functor $F$ : Set $\rightarrow$ Set preserves weak pullbacks if applying $F$ to a weak pullback $(P, k: P \rightarrow S, l: P \rightarrow U)$, of functions $f: S \rightarrow T$ and $g: U \rightarrow T$, yields again a weak pullback: $(F(P), F(k): F(P) \rightarrow F(S), F(l): F(P) \rightarrow F(U))$, now of the functions $F(f): F(S) \rightarrow F(T)$ and $F(g): F(U) \rightarrow F(T)$. 
We show that $\mathcal{D}$ preserves weak pullbacks. Let $(P, k: P \rightarrow S, l: P \rightarrow U)$ be a weak pullback of functions $f: S \rightarrow T$ and $g: U \rightarrow T$. Let $\mu$ in $\mathcal{D}(S)$ and $\nu$ in $\mathcal{D}(U)$ be two simple probability distributions with $\mathcal{D}(f)(\mu)=\mathcal{D}(g)(\nu)$. We have to establish the existence of a distribution $\pi$ in $\mathcal{D}(P)$ such that $\mathcal{D}(k)(\pi)=\mu$ and $\mathcal{D}(l)(\pi)=\nu$. Let the finite support of $\mu$ and $\nu$ be given by

$$
S_{0}=\{s \in S \mid \mu(s)>0\}, \quad U_{0}=\{u \in U \mid \nu(u)>0\} .
$$

It follows from $\mathcal{D}(f)(\mu)=\mathcal{D}(g)(\nu)$ that $f\left(S_{0}\right)=g\left(U_{0}\right)$ : For the inclusion from left to right, consider $f(s)$, for $s$ in $S_{0}$. Because

$$
\nu\left[g^{-1}(\{f(s)\}]=\mathcal{D}(g)(\nu)(f(s))=\mathcal{D}(f)(\mu)(f(s))=\mu\left[f^{-1}(\{f(s)\})\right] \geq \mu(s)>0,\right.
$$

there exists $u$ in $U_{0}$ with $g(u)=f(s)$. Thus $f\left(S_{0}\right) \subseteq g\left(U_{0}\right)$. The reverse follows by symmetry. It follows from $f\left(S_{0}\right)=g\left(U_{0}\right)$ and the fact that $P$ is a weak pullback that

$$
P^{\prime}=\left\{p \in P \mid \exists s \in S_{0} \exists u \in U_{0}, f(s)=g(u) \text { and } k(p)=s \text { and } l(p)=u\right\}
$$

is a subset of $P$ with $k\left(P^{\prime}\right)=S_{0}$ and $l\left(P^{\prime}\right)=U_{0}$. Let $P_{0}$ be a finite subset of $P^{\prime}$ obtained by removing 'duplicates', where $p$ is a duplicate of $p^{\prime}$ if $k(p)=k\left(p^{\prime}\right)$ and $l(p)=l\left(p^{\prime}\right)$. Then also $k\left(P_{0}\right)=S_{0}$ and $l\left(P_{0}\right)=U_{0}$.

We shall next use the max-flow min-cut theorem from graph theory to establish the existence of a simple probability distribution $\pi: P \rightarrow[0,1]$ with support in $P_{0}$, such that $\mathcal{D}(k)(\pi)=\mu$ and $\mathcal{D}(l)(\pi)=\nu$. (See also [Mos97], where the same functor $\mathcal{D}$ is considered, for a proof 'from first principles'.) Let us first briefly recall this theorem (for further details see, for instance, [Bol79, p47]). Let $\mathcal{G}=\langle\mathcal{N}, \mathcal{E}\rangle$ be a finite directed graph with nodes $\mathcal{N}$ and edges $\mathcal{E} \subseteq \mathcal{N} \times \mathcal{N}$. Let 0 and 1 be two nodes in $\mathcal{N}$ called source and sink. A flow from 0 to 1 is any function $d: \mathcal{E} \rightarrow[0,1]$ such that for all nodes $x$ different from 0 and 1 :

$$
\sum_{y \in \Gamma^{+}(x)} f(x, y)=\sum_{z \in \Gamma^{-}(x)} f(z, x),
$$

where $\Gamma^{+}(x)=\{y \in \mathcal{N} \mid\langle x, y\rangle \in \mathcal{E}\}, \Gamma^{-}(x)=\{z \in \mathcal{N} \mid\langle z, x\rangle \in \mathcal{E}\}$. The value of a flow $d$ is given by

$$
\sum_{y \in \Gamma^{+}(0)} f(0, y)-\sum_{y \in \Gamma^{-}(0)} f(y, 0) .
$$

The flow through any edge will be bounded by its capacity, which is specified by a function $c: \mathcal{E} \rightarrow[0,1]$. A cut is a subset $N \subseteq \mathcal{N}$ with $0 \in N$ and $1 \notin N$. The capacity of a cut $N$ is defined as

$$
\sum\{c(\langle x, y\rangle) \mid x \in N \text { and } y \notin N\} .
$$

It is easy to see that there exists a cut whose capacity is minimal, and that there exists a flow with maximal value. Also that the value of this maximal flow is at most the value of the minimal cut. The max-flow min-cut theorem says that this trivial inequality is, in fact, an equality.

Next consider the finite directed graph $\mathcal{G}=\langle\mathcal{N}, \mathcal{E}\rangle$ with nodes $\mathcal{N}=\{0,1\} \cup S_{0} \cup U_{0}$ and edges $\mathcal{E}=\left\{E_{x} \mid x \in S_{0} \cup U_{0} \cup P_{0}\right\}$, where $E_{s}=\langle 0, s\rangle, E_{u}=\langle u, 1\rangle$, and $E_{p}=\langle k(p), l(p)\rangle$, for $s$ in $S_{0}, u$ in $U_{0}$, and $p$ in $P_{0}$, respectively. (We assume $S_{0}, U_{0}, P_{0}$ to be disjoint.) Note that for all $s \in S_{0}$ there exists $u \in U_{0}$ and an edge $\langle s, u\rangle \in \mathcal{E}$, and vice versa. Let the capacity $c: \mathcal{E} \rightarrow[0,1]$ be defined by $c\left(E_{s}\right)=\mu(s), c\left(E_{u}\right)=\nu(u)$, and $c\left(E_{p}\right)=1$. 
The capacity of the cut $N=\{0\}$ is $\Sigma\left\{\mu(s) \mid s \in S_{0}\right\}=1$. If we try to obtain a cut whose value is smaller than 1 by including $s$ in $N$, for some $s$ in $S_{0}$-thus trying to reduce the capacity by the value $\mu(s)$ - then we are forced to include all $u \in g^{-1}(\{f(s)\})$ as well, since for any such $u$ there exists $p \in P_{0}$ with $k(p)=s$ and $l(p)=u$, and hence an edge $\langle s, u\rangle$ with capacity 1 . For the possible reduction by $\mu(s)$ we thus obtain an addition of $\nu\left[g^{-1}(\{f(s)\})\right]$ which is, as we have seen above, at least $\mu(s)$. By a similar argument one can show that adding any $u$ in $U_{0}$ to $N$ will not reduce its capacity. It follows that $N$ is a minimal cut.

By the max-flow min-cut theorem, there exists a flow function $d: \mathcal{E} \rightarrow[0,1]$ with (maximal) value 1. It follows (from the definition of flow function) that $d\left(E_{s}\right)=\mu(s)$ and $d\left(E_{u}\right)=\nu(u)$. As a consequence, defining $\pi: P \rightarrow[0,1]$ by $\pi(p)=d\left(E_{p}\right)$, if $p \in P_{0}$, and 0 , otherwise, gives us the distribution we are looking for: The function $\pi$ is a simple probability distribution $\left(P_{0}\right.$ is finite, $\pi\left[P_{0}\right]=1$ ), and, for $s$ in $S_{0}$,

$$
\begin{aligned}
& \mathcal{D}(k)(\pi)(s)=\pi\left[k^{-1}(\{s\})\right]=\sum\left\{\pi(p) \mid p \in P_{0} \text { and } k(p)=s\right\} \\
& \quad=\sum\left\{d\left(E_{p}\right) \mid p \in P_{0} \text { and } k(p)=s\right\}=d\left(E_{s}\right)=\mu(s) .
\end{aligned}
$$

So $\mathcal{D}(k)(\pi)=\mu$. Similarly one shows that $\mathcal{D}(l)(\pi)=\nu$. We conclude that the simple distribution $\pi$ has the desired property and that $\mathcal{D}$ preserves weak pullbacks. 